\title{
Predictors of Solid Organ Injury Following Blunt Abdominal Trauma: A Single Institution Process Improvement Initiative
}

Keywords: Trauma; Solid organ injury; Predic tors

\begin{abstract}
Background: Computed tomography (CT) scan remains the gold standard for identifying solid organ injuries (SOI) following blunt abdominal trauma but subjects the child to ionizing radiation, requires transportation out of the emergency department, may require sedation, and has a significant cost.
\end{abstract}

Methods: We performed a 5-year retrospective review of a prospectively collected trauma database at a Level One Pediatric Trauma Center to determine the best screening tools for identifying SOI prior to obtaining a CT scan.

Results: Forty-three patients with SOI (paired with 47 agematched controls) were included. Univariate logistic regression analysis demonstrated the following factors to be predictive of $\mathrm{SO}$ : complaint of abdominal pain $(p=0.021)$; tenderness on abdominal exam ( $p=0.001)$; and abnormal lipase $(p=0.013)$, AST ( $p=0.037)$, or ALT $(p=0.001)$. Vital signs, hemoglobin, amylase, and alkaline phosphatase did not predict SOI. On multivariate analysis abdominal tenderness, abnormal lipase, and abnormal ALT remained significant ( $p$-values $0.006,0.049$, and 0.002 , respectively)

Conclusions: In this single institution process improvement initia tive, not all laboratory studies routinely obtained in injured patients predicted SOI. Physical exam remains an important part of the trauma evaluation. Utilizing this data, we can devise a more cost effective method to screen for SOI and limit CT scan use in injured children.

\section{Background}

At least 7.5 million emergency department visits a year in the United States are related to traumatic injuries in children [1], and trauma remains the most common cause of childhood morbidity and mortality $[2,3]$. While they play a significant role in death and disability following trauma, intra-abdominal solid organ injuries (SOI) can be difficult to identify [2]. Medical personnel who care for pediatric trauma patients have 3 major modalities available during evaluation of potential SOI: physical examination, laboratory tests, and abdominopelvic computed tomography (CT) scan. While many agree that the physical exam, looking for both tenderness and abdominal wall abrasions and/or bruising, is the cornerstone of evaluation [3-5], it may be difficult to evaluate very young children or those who are non-verbal or have an altered level of consciousness $[2,3]$.

Laboratory testing, while routinely performed, has questionable utility based on numerous available studies [3,6-11]. Holmes et al. make the point that laboratory studies are only a useful adjunct when interpreted along with physical exam findings [3]. CT scan of the abdomen and pelvis is the gold standard imaging modality for identifying solid organ injury after blunt abdominal trauma $[3,6]$.

\section{Journal of Surgery}

Alia Whitehead ${ }^{1}$, Matthew Moront ${ }^{1}$, Lezhou $\mathbf{W u}^{2}$, and Rajeev Prasad ${ }^{1 *}$

${ }^{1}$ Department of Pediatric General, Thoracic, and Minimally Invasive Surgery, St. Christopher's Hospital for Children/Drexel University College of Medicine, Philadelphia, PA

${ }^{2}$ Department of Statistics, Drexel University, Philadelphia, PA, USA

\section{*Address for Correspondence}

Rajeev Prasad, MD, Department of Pediatric General, Thoracic, and Minimally Invasive Surgery, St. Christopher's Hospital for Children/ Drexel University College of Medicine, 3601 A Street, Philadelphia, PA 19134, USA, Tel: 215-427-5446; Fax: 215-762-4616; E-mail: rajeev.prasad@tenethealth.com

Copyright: (c) 2015 Whitehead A, et al. This is an open access article distributed under the Creative Commons Attribution License, which permits unrestricted use distribution, and reproduction in any medium, provided the original work is properly cited.

Submission: 22 November 2014

Accepted: 31 December 2014

Published: 05 January 2015

Reviewed \& Approved by: Dr. Joseph A locono, Associate Professor of Surgery and Pediatrics, Division Chief of Pediatric Surgery, Kentucky Children's Hospital, University of Kentucky, USA

Unfortunately, an abdominopelvic CT scan subjects the child to a substantial amount of ionizing radiation, requires transportation out of the emergency department, may require sedation, and has a significant cost [3].

In our institution patients routinely undergo abdominal CT scan in the presence of isolated laboratory value abnormalities including only modest elevations in transaminases, abnormally low hemoglobin, or mildly elevated pancreatic enzymes. We set out to systematically review our practices and protocols utilized in trauma patients in our institution with the goal to determine which steps in the trauma evaluation were most important in predicting SOI and therefore the most important in aiding in the decision of when to order an abdominopelvic CT scan.

\section{Methods}

We conducted a retrospective chart review of a prospectively collected trauma database at a Level One Pediatric Trauma Center from February 2009 to September 2013. Our study group consisted of all patients with an intra-abdominal SOI (liver, spleen, pancreas, kidney, or multiple organs) following blunt trauma. Patients having sustained penetrating trauma were excluded. Our control group consisted of a random sampling of patients from the same time period who underwent a CT scan of the abdomen and pelvis for blunt trauma but did not have an intra-abdominal injury. Data collected included patient age, mechanism of injury, subjective patient complaints, vital signs, details of the abdominal exam, routinely obtained trauma panel laboratory values, and finally the type(s) of injury eventually confirmed by abdominopelvic CT scan.

All statistical analyses were conducted using SAS software, version 9.2 (SAS Institute, Inc., Cary, North Carolina). To explore the association of SOI with a variety of clinical indicators, univariate and multivariate logistic regression models were applied, respectively; the corresponding odds ratios (OR) and 95\% confidence intervals (CI) were then reported. Ultimately, we developed an organ-specific logistic regression model for multiple organ, hepatic, splenic, and 
Citation: Whitehead A, Moront M, Wu L, Prasad R. Predictors of Solid Organ Injury Following Blunt Abdominal Trauma: A Single Institution Process Improvement Initiative. J Surgery. 2015;3(1): 3.

ISSN: $2332-4139$

renal injuries. Because of the small sample size, organ-specific analysis was not performed for pancreatic injury. A p-value less than 0.05 was considered statistically significant.

\section{Results}

Baseline characteristics of the study population are presented in Table 1 . The two study groups were matched with regard to age $(\mathrm{p}=0.833)$, mechanism of injury $(\mathrm{p}=0.362)$, and GCS $(\mathrm{p}=0.92)$. Forty-three patients suffered 51 SOI (i.e., some patients had multiorgan injury). Univariate logistic regression analysis (Table 2) demonstrated that the subjective complaint of abdominal pain by a patient statistically predicted an intra-abdominal SOI. A normal abdominal examination predicted the absence of SOI (odds ratio

Table 1: Baseline characteristics of study population.

\begin{tabular}{|c|c|c|c|c|}
\hline & Total $(n=90)$ & $\begin{array}{c}\text { Control } \\
(n=47)\end{array}$ & $\begin{array}{c}\text { Injured } \\
(n=43)\end{array}$ & P-value \\
\hline Median Age (years) & 7.0 & 7.0 & 6.0 & 0.833 \\
\hline \multicolumn{5}{|l|}{ Length of Stay } \\
\hline 1 day & 13 & 13 & 0 & \\
\hline 2 days & 10 & 9 & 1 & \\
\hline 3 days & 14 & 7 & 7 & \\
\hline 4 days & 17 & 5 & 12 & \\
\hline 5 days & 10 & 5 & 5 & \\
\hline 6 days & 8 & 2 & 6 & \\
\hline 7 days & 6 & 2 & 4 & \\
\hline$>$ or $=8$ days & 12 & 4 & 8 & \\
\hline Mechanism of Injury & & & & 0.362 \\
\hline Assault & 2 & 2 & 0 & \\
\hline Auto vs. Pedestrian & 42 & 25 & 17 & \\
\hline Fall & 20 & 7 & 13 & \\
\hline MVC & 5 & 3 & 2 & \\
\hline $\begin{array}{l}\text { Non-Accidental } \\
\text { Trauma }\end{array}$ & 14 & 6 & 8 & \\
\hline Car vs. Bike & 1 & 1 & 0 & \\
\hline TV Tip over & 1 & 1 & 0 & \\
\hline Other & 5 & 2 & 3 & \\
\hline \multicolumn{5}{|l|}{ Grade of Injury (Liver) } \\
\hline 1 & 3 & 0 & 3 & \\
\hline 2 & 7 & 0 & 7 & \\
\hline 3 & 11 & 0 & 11 & \\
\hline 4 & 4 & 0 & 4 & \\
\hline 5 & 1 & 0 & 1 & \\
\hline NA & 2 & 0 & 2 & \\
\hline \multicolumn{5}{|l|}{ Grade of Injury (Spleen) } \\
\hline 1 & 4 & 0 & 4 & \\
\hline 2 & 3 & 0 & 3 & \\
\hline 3 & 3 & 0 & 3 & \\
\hline 4 & 2 & 0 & 2 & \\
\hline 5 & 1 & 0 & 1 & \\
\hline \multicolumn{5}{|l|}{ Grade of Injury (Kidney) } \\
\hline 1 & 1 & 0 & 1 & \\
\hline 5 & 2 & 0 & 2 & \\
\hline NA & 2 & 0 & 2 & \\
\hline \multicolumn{5}{|l|}{$\begin{array}{c}\text { Grade of Injury } \\
\text { (Pancreas) }\end{array}$} \\
\hline 2 & 2 & 0 & 2 & \\
\hline NA & 3 & 0 & 3 & \\
\hline
\end{tabular}

*NA: Not Available
Table 2: Unadjusted odds ratio for intra-abdominal solid organ injury.

\begin{tabular}{|c|c|c|c|}
\hline & Odds ratio & $95 \% \mathrm{Cl}$ & P- value \\
\hline Complaint of Abdominal Pain & 3.040 & $1.180-7.829$ & 0.021 \\
\hline Normal Abdominal Exam & 0.313 & $0.130-0.754$ & 0.010 \\
\hline Abdominal Tenderness & 4.856 & $1.893-12.453$ & 0.001 \\
\hline Abdominal Distention & 1.414 & $0.354-5.650$ & 0.624 \\
\hline Abdominal Guarding & 3.450 & $0.345-034.497$ & 0.292 \\
\hline Abdominal Bruising & 1.687 & $0.268-10.617$ & 0.577 \\
\hline Abdominal Abrasion & 0.715 & $0.114-4.501$ & 0.721 \\
\hline Heart Rate & 1.538 & $0.665-3.558$ & 0.315 \\
\hline Respiratory Rate & 0.685 & $0.222-2.115$ & 0.510 \\
\hline Systolic BP & 0.956 & $0.390-2.345$ & 0.922 \\
\hline Diastolic BP & 0.956 & $0.390-2.345$ & 0.922 \\
\hline WBC & 1.684 & $0.725-3.916$ & 0.226 \\
\hline $\mathrm{Hgb}$ & 0.590 & $0.256-1.361$ & 0.216 \\
\hline Platelets & 1.743 & $0.457-6.553$ & 0.416 \\
\hline Amylase & 2.286 & $0.794-6.580$ & 0.125 \\
\hline Lipase & 4.707 & $1.395-15.887$ & 0.013 \\
\hline AST & 2.914 & $1.067-7.959$ & 0.037 \\
\hline ALT & 6.138 & $2.053-18.357$ & 0.001 \\
\hline Alkaline Phosphatase & 1.920 & $0.576-6.400$ & 0.288 \\
\hline Urinalysis (RBCs) & 1.647 & $0.657-4.126$ & 0.287 \\
\hline
\end{tabular}

*Univariate logistic regression model

0.313 (95\% CI 0.130-0.754). Also, the physical finding of tenderness on examination predicted SOI in our study group. The findings of distension, guarding, bruising, and abrasions on exam did not predict SOI. Abnormal vital signs did not predict SOI in our patients. Abnormal values for white blood cell count, hemoglobin, platelets, amylase, alkaline phosphatase, and red blood cells in the urine did not predict SOI in the univariate model. However, an abnormal lipase, AST, or ALT were all statistically significantly associated with SOI (all $\mathrm{P}<0.05)$ in univariate analysis.

Further multivariate analysis (Table 3) showed that patients with intra-abdominal SOI were statistically more likely to have abdominal tenderness on examination ( $\mathrm{p}=0.006$, OR 18.135 , 95\% CI 2.298 143.085) as compared to controls. An abnormal lipase ( $\mathrm{p}=0.049$, OR 5.191, 95\% CI 1.008-26.735), ALT ( $\mathrm{p}=0.002$, OR 17.273, 95\% CI 2.773-107.607), and alkaline phosphatase ( $\mathrm{p}=0.002$, OR $25.219,95 \%$ CI 3.217-197.705) were associated with increased risk of SOI.

In organ-specific injury analysis (Table 4), only abnormally elevated levels of ALT ( $\mathrm{p}=0.012$, OR 17.488, 95\%CI 1.857-164.710) were associated with liver injury (the most common type of injury noted during the study period). Elevated lipase levels predicted multiple organ ( $\mathrm{p}=0.009$, OR 27.750, 95\%CI 2.307-333.763), splenic $(\mathrm{p}=0.004$, OR 18.500, 95\%CI 2.538-134.834), and renal injuries ( $\mathrm{p}=0.013$, OR 13.876, 95\%CI 1.759-109.444). Notably, confidence levels were wide, confirming that the sample size was limited.

\section{Discussion}

According to Rothrock et al., indications for abdominopelvic CT scan after blunt trauma are: 1) abdominal pain or tenderness, 
Citation: Whitehead A, Moront M, Wu L, Prasad R. Predictors of Solid Organ Injury Following Blunt Abdominal Trauma: A Single Institution Process Improvement Initiative. J Surgery. 2015;3(1): 3.

ISSN: 2332-4139

Table 3: Adjusted odds ratio for intra-abdominal solid organ injury.

\begin{tabular}{|l|c|c|c|}
\hline & Odds ratio & $95 \% \mathbf{C l}$ & P-value \\
\hline Abdominal Tenderness & 18.135 & $2.298-143.085$ & 0.006 \\
\hline Lipase & 5.191 & $1.008-26.735$ & 0.049 \\
\hline ALT & 17.273 & $2.773-107.607$ & 0.002 \\
\hline Alkaline Phosphatase & 25.219 & $3.217-197.705$ & 0.002 \\
\hline
\end{tabular}

*Multivariate logistic regression model

Table 4: Organ-specific injury analysis.

\begin{tabular}{|l|l|l|}
\hline & OR $(\mathbf{9 5} \% \mathbf{C l})$ & P-value \\
\hline Multiple organ injury $(\mathbf{n}=\mathbf{6})$ & & \\
\hline Lipase & $27.750(2.307-333.763)$ & 0.009 \\
\hline Liver injury $(\mathbf{n = 2 8 )}$ & & \\
\hline ALT & $17.488(1.857-164.710)$ & 0.012 \\
\hline Spleen injury $(\mathbf{n}=13)$ & & \\
\hline Lipase & $18.500(2.538-134.834)$ & 0.004 \\
\hline Kidney injury $(\mathbf{n}=5)$ & & \\
\hline Lipase & $13.876(1.759-109.444)$ & 0.013 \\
\hline
\end{tabular}

2) fluid requirement without obvious blood loss, 3) known multisystem trauma, 4) altered mental status, 5) hemoglobin $<10$ without obvious blood loss, and 6) minor injury with gross hematuria (or $\geq 50 \mathrm{RBC} / \mathrm{hpf}$ ), although the exact threshold is controversial [6]. In our single institution study, however, we found that most laboratory studies routinely obtained in trauma patients are not predictive of intra-abdominal SOI. In multivariate analysis, only elevated lipase, ALT, and alkaline phosphatase were predictive of solid organ injury. A surprising finding in our cohort of patients was that abnormal hemoglobin was not predictive of SOI; others would argue the contrary [6]. We feel that this finding may be due to a lack of severity of the injuries seen in this time period as greater than $75 \%$ of all liver and spleen injuries in our cohort were grade 3 or lower.

Although they were not helpful in predicting SOI in this study, we contend that vital signs will always remain an important part of the trauma evaluation. It is possible that abnormal vital signs indicative of hemodynamic instability, such as tachycardia, were masked by patients who were tachycardic due to anxiety or pain, a common occurrence in the pediatric population. Furthermore, as stated above, the injuries in our study group tended to be Grade 3 or lower, suggesting a less severe degree of blood loss in these patients.

While it was not significant in multivariate analysis, our study suggests that a patient's subjective complaint of abdominal pain should prompt further evaluation. Glasgow Coma Scales for the control and injured groups were relatively normal (13.9 and 14, respectively) so we believe that this was a valid measure in our study. Obviously a child with altered mental status would not be a reliable historian, however, and may require imaging. Tenderness on physical examination remains the most important part of the trauma evaluation, as compared to guarding, bruising or abrasion in most cases, and is a reason to obtain abdominal imaging. Again, this may be difficult to elicit in the child with altered mental status. A concurrent thoracic injury, which was insignificant on multivariate analysis of our data, may also be a reason for imaging of the abdomen and pelvis, given the amount of force generally required to cause a significant injury of the chest in a child.

In conclusion, the complaint of abdominal pain and the ability to elicit tenderness on examination are significant findings during the trauma assessment and should prompt the pediatric trauma provider to consider further imaging with abdominopelvic CT scan in children with blunt abdominal trauma. An abnormal lipase predicts not only pancreatic injury but also splenic and renal injury likely given the close proximity of these organs to one another. Finally, elevated transaminases (specifically ALT) and alkaline phosphatase represent the most important components of the trauma laboratory panel for predicting hepatic injury. Although not statistically significant in our study due to lower grades of injury and, possibly, small sample size, we suggest that abnormal vital signs, an abnormally low hemoglobin, and a concurrent major thoracic injury should still alert the provider of possible significant intra-abdominal SOI.

Utilizing our data, we can devise a potentially more cost effective and safer approach to screen for SOI in children with blunt abdominal trauma by streamlining the routine trauma laboratory panel and limiting CT scan use.

\section{References}

1. Hom J (2010) The risk of intra-abdominal injuries in pediatric patients with stable blunt abdominal trauma and negative abdominal computed tomography. Acad Emerg Med 17: 469-475.

2. Wegner S, Colletti JE, Van Wie D (2006) Pediatric blunt abdominal trauma Pediatr Clin North Am 53: 243-256.

3. Holmes J, Sokolove PE, Brant WE, Palchak MJ, Vance CW, et al. (2002) Identification of children with intra-abdominal injuries after blunt trauma. Ann Emerg Med 39: 500-509.

4. Sokolove PE, Kupperman N, Holmes JF (2005) Association between the "seat belt sign" and intra-abdominal injury in children with blunt torso trauma. Acad Emerg Med 12: 808-813.

5. Lutz N, Nance ML, Kallan MJ, Arbogast KB, Durbin DR, et al. (2004) Incidence and clinical significance of abdominal wall bruising in restrained children involved in motor vehicle crashes. J Pediatr Surg 39: 972-975

6. Rothrock SG, Green SM, Morgan R (2000) Abdominal trauma in infants and children: prompt identification and early management of serious and lifethreatening injuries. Part 1: Injury patterns and initial assessment. Pediatr Emerg Care 16: 106-115.

7. Adamson WT, Hebra A, Thomas PB, Wagstaff P, Tagge EP, et al. (2003) Serum amylase and lipase alone are not cost-effective screening methods for pediatric pancreatic trauma. J Pediatr Surg 38: 354-357.

8. Herman R, Guire KE, Burd RS, Mooney DP, Ehlrich PF (2011) Utility of amylase and lipase as predictors of grade of injury or outcomes in pediatric patients with pancreatic trauma. J Pediatr Surg 46: 923-926

9. Capraro AJ, Mooney D, Waltzman ML (2006) The use of routine laboratory studies as screening tools in pediatric abdominal trauma. Pediatr Emerg Care 22: $480-484$.

10. Isaacman DJ, Scarfone RJ, Kost SI, Gochman RF, Davis HW, et al. (1993) Utility of routine laboratory testing for detecting intra-abdominal injury in the pediatric trauma patient. Pediatrics 92: 691-694.

11. Ford EG, Karamanoukian HL, McGrath N, Mahour GH (1990) Emergency center laboratory evaluation of pediatric trauma victims. Am Surg 56: 752 757. 